\title{
Simultaneous image fusion and denoising by using fractional-order gradient information
}

\author{
Mei, Jin Jin; Dong, Yiqiu; Huang, Ting Zhu
}

Published in:

Journal of Computational and Applied Mathematics

Link to article, DOI:

10.1016/j.cam.2018.11.012

Publication date:

2019

Document Version

Peer reviewed version

Link back to DTU Orbit

Citation $(A P A)$ :

Mei, J. J., Dong, Y., \& Huang, T. Z. (2019). Simultaneous image fusion and denoising by using fractional-order gradient information. Journal of Computational and Applied Mathematics, 351, 212-227. https://doi.org/10.1016/j.cam.2018.11.012

\section{General rights}

Copyright and moral rights for the publications made accessible in the public portal are retained by the authors and/or other copyright owners and it is a condition of accessing publications that users recognise and abide by the legal requirements associated with these rights.

- Users may download and print one copy of any publication from the public portal for the purpose of private study or research.

- You may not further distribute the material or use it for any profit-making activity or commercial gain

- You may freely distribute the URL identifying the publication in the public portal 


\title{
Simultaneous Image Fusion and Denoising by Using Fractional-Order Gradient Information *
}

\author{
Jin-Jin Mei $\quad$ Yiqiu Dong ${ }^{\dagger} \quad$ Ting-Zhu Huang ${ }^{\S}$
}

\begin{abstract}
Image fusion and denoising are significant in image processing because of the availability of multi-sensor and the presence of the noise. The first-order and second-order gradient information have been effectively applied to deal with fusing the noise-free source images. In this paper, we utilize the fractional-order derivatives to represent image features, and propose two new convex variational models for fusing noisy source images. Furthermore, we apply an alternating direction method of multiplier (ADMM) to solve the minimization problems in the proposed models. Numerical experiments show that the proposed methods outperform the conventional total variation methods for simultaneously fusing and denoising.

Keywords: image fusion and denoising, alternating direction method of multiplier, inverse problem, fractional-order derivative, structure tensor
\end{abstract}

\section{Introduction}

Image fusion is to synthesize several source images of the same scene into a single image which contains much more visual information. It is an important issue due to the availability of multisensor data in various fields such as remote sensing, medical diagnosis, defect inspection, and military surveillance [1-3]. But, the observed source images are inevitably corrupted by the noise during the process of image collection, acquisition, transmission and storage. In order to obtain a high-quality image, it is necessary to simultaneously denoise and fuse the source images.

Before introducing the fusion methods, we first set up some notations. Suppose that $\Omega \subset \mathbb{R}^{2}$ is a connected bounded image domain with the compacted Lipschitz boundary, $f_{i}: \Omega \rightarrow \mathbb{R}$, $i=1,2, \cdots, l$ are the source images, and $u: \Omega \rightarrow \mathbb{R}$ is the unknown fused image. In this paper, we mainly focus on the pixel-level fusion methods, i.e., the methods to estimate the value for each point in the fused image based on several source images through a feature selection rule [4-7]. Generally, the pixel-level fusion methods mainly consist of spatial domain fusion methods [8-12] and transform domain fusion methods [13-19]. Among the spatial domain fusion methods, several variational methods based on fusing the gradient information have been studied, see e.g. $[11,12,20-23]$.

${ }^{*}$ This research was supported by the National Science Foundation of China $(61772003,11701388)$, and the Fundamental Research Funds for the Central Universities (ZYGX2016J132).

${ }^{\dagger}$ School of Mathematics and Statistics, Fuyang Normal University, Fuyang 236037, Anhui, P. R. China. School of Mathematical Sciences, University of Electronic Science and Technology of China, Chengdu 611731, P. R. China ( meijinjin666@126.com).

${ }^{\ddagger}$ College of Mathematics and Statistics, Shenzhen University, Shenzhen, Guangdong, P. R. China. Department of Applied Mathematics and Computer Science, Technical University of Denmark, 2800 Kgs. Lyngby, Denmark (yido@dtu.dk).

${ }^{\S}$ School of Mathematical Sciences, University of Electronic Science and Technology of China, Chengdu 611731, P. R. China (tingzhuhuang@126.com). 
In $[21,22]$, the structure tensor is used for depicting the local structure feature, which is defined as follows

$$
G(x, y):=\left(\begin{array}{ll}
\sum_{i=1}^{l}\left(\frac{\partial f_{i}}{\partial x}\right)^{2} & \sum_{i=1}^{l} \frac{\partial f_{i}}{\partial x} \frac{\partial f_{i}}{\partial y} \\
\sum_{i=1}^{l} \frac{\partial f_{i}}{\partial y} \frac{\partial f_{i}}{\partial x} & \sum_{i=1}^{l}\left(\frac{\partial f_{i}}{\partial y}\right)^{2}
\end{array}\right), \quad \forall(x, y) \in \Omega,
$$

where $\frac{\partial f}{\partial x}$ and $\frac{\partial f}{\partial y}$ give the derivatives of the function $f$ along $x$ - and $y$-direction, respectively. Since $G$ is positive semi-definite, the target gradient $V: \Omega \rightarrow \mathbb{R}^{2}$ is formulated as

$$
V:=\sqrt{\lambda} \mathbf{e}
$$

where $\lambda$ denotes the largest eigenvalue of $G$ that conveys the sharp information, $\mathbf{e}$ is the corresponding unit eigenvector which indicates the orientation maximizing the pixel-value fluctuations and satisfies that $\left\langle\mathbf{e}, \sum_{i=1}^{l} \nabla f_{i}\right\rangle \geqslant 0$. Then, the variational model for obtaining the fused image $u$ is proposed as

$$
\min _{u} \int_{\Omega}|\nabla u-V|^{2} d x
$$

Note that the model (1) results in loss of contrast and the model solution is not unique. In [11], Piella combined the image enhancement techniques and proposed a variational model for integrating the salient structure of source images and enhancing the contrast of the fused image. The author derived the corresponding Euler-Lagrange equation and applied the gradient descent method for obtaining the unknown fused image. But due to the slow convergent speed, the gradient descent method is extremely time-consuming. In [23], the authors proposed a new variational model by fusing the first- and second-order gradient information (called as FSG for short):

$$
\min _{u} \int_{\Omega}\left(|\nabla u-s v|+\alpha\left|\nabla^{2} u-s w\right|\right) d x+\frac{\beta}{2} \int_{\Omega}\left|u-u_{0}\right|^{2} d x
$$

where $u_{0}$ is a pre-defined image, $\alpha \geqslant 0, \beta>0, s \geqslant 1, v$ and $w$ denote the target gradients which integrated the first- and second-order gradient information from the source images, respectively. A new feature selection was used to construct the target gradient $v$ and $w$. Note that if $s=1$, the model (2) is just for image fusion; if $s>1$, the model is for simultaneous image fusion and enhancement. Moreover, the split Bregman method [24-26] was applied to solve the model (2).

As far as we know, only a little work was done for fusing the noisy source images. In [20], Wang et al. proposed a weighted variational method based on the total variation (TV) regularization (called as WTV for short), and the corresponding variational model is

$$
\min _{u} \int_{\Omega}\left(\alpha_{1}(x)\left(u-f_{1}\right)^{2}+\alpha_{2}(x)\left(u-f_{2}\right)^{2}\right) d x+2 \beta \int_{\Omega}|D u|
$$

where $\alpha_{1}(x)$ and $\alpha_{2}(x)$ are two nonnegative weight functions and satisfy $\alpha_{1}(x)+\alpha_{2}(x)=1$, and $\beta>0$ is the regularization parameter that trades off the fit to the noisy source images and the smoothness from TV. The gradient descent method is applied to solve the minimization problem in (3). In [27], the authors proposed an adaptive sparse representation model for image fusion and denoising. Assume that the high-quality image patches were classified into several categories in advance. Then, based on the gradient information from the source images, they learned a set of compacted sub-dictionaries from numerous high-quality image patches and designed an algorithm to select adaptively one from the sub-dictionaries. This method is a patch-based dictionary learning method, and in order to learn the dictionaries it requires some extra computations comparing with simple gradient-based variational methods such as WTV. 
Recently, in [28] the theoretical properties of the fractional-order derivatives were studied. It is shown that by incorporating the fractional-order derivatives as the regularization of the variational method can preserve sharp edges and keep smoothness in homogeneous regions. Inspired by this advantage, in this paper we combine the fractional-order variation and propose two novel variational models for image fusion and denoising. We first build a target fractional-order gradient based on the noisy source images according to a simpler salience criterion than the one introduced in [23]. Then, we require that the fractional-order gradient of the fused image should be matched with the target fractional-order gradient in the sense of $L^{2}$ norm or $L^{1}$ norm in order to obtain the fused image. Meanwhile, we set a data-fitting term to ensure the fused image close to a pre-defined image. Since the source images are corrupted by the noise, we add the TV regularization into our variational models in order to reduce the influence from the noise. For the new variational models, we prove the existence and uniqueness of the solution. In addition, under the framework of the alternating direction method of multipliers (ADMM) [29-31], a numerical algorithm is developed for solving the minimization problems in the proposed models. The numerical results indicate that our methods have the potential to outperform the other gradient-based variational methods.

In conclude, our main contributions are summarized as follows:

1. A target fractional-order gradient from the noisy source images is constructed through a simplified salience criterion.

2. Two new variational models for simultaneously fusing and denoising are proposed, and the existence and uniqueness to the solution for the new models are studied.

3. The ADMM algorithm is applied to solve the minimization problems in the new models.

4. The performance of the new methods are studied through many numerical experiments.

The rest of the paper is organized as follows. In the next section, we review the elementary properties of the fractional-order derivative. In Section 3, we introduce a method to obtain the target fractional-order feature from the noisy source images and propose two new variational models with the TV regularization for image fusion and denoising. Meanwhile, we prove the existence and the uniqueness to the solution for the new models. In Section 4, we apply the ADMM algorithm to solve the minimization problems in the proposed models. Numerical experiments are shown in Section 5. Finally, we conclude the paper in Section 6.

\section{Fractional-order derivative}

Recently, the fractional-order derivative was used in several image processing tasks, see $[28,32$ 34]. For self-containedness of the paper, in this section we briefly review the definitions and some properties of the fractional-order derivative.

As a generalization of the integer-order derivatives, the definitions and properties of the fractionalorder derivative have been studied over the one-dimensional domain, see [35-37] and references therein. Assume that a fraction $\gamma \in \mathbb{R}^{+}$and a function $h \in L^{1}([a, b] ; \mathbb{R})$. The left and right Riemann-Liouville (RL) fractional-order derivatives of $h$ are respectively defined as

$$
\begin{array}{lll}
{ }_{a} D_{x}^{\gamma}[h](x) & =\frac{1}{\Gamma(1-\gamma)} \frac{d}{d x} \int_{a}^{x} \frac{h(\tau)}{(x-\tau)^{\gamma}} d \tau, & x \in(a, b] \\
{ }_{x} D_{b}^{\gamma}[h](x) & =\frac{-1}{\Gamma(1-\gamma)} \frac{d}{d x} \int_{x}^{b} \frac{h(\tau)}{(\tau-x)^{\gamma}} d \tau, & x \in[a, b)
\end{array}
$$


where $\Gamma(\gamma)$ represents a Gamma function. Based on the left and the right RL fractional-order derivatives, the Riesz-RL (central) fractional-order derivative is given by

$$
{ }_{a} D_{b}^{\gamma}[h](x)=\frac{1}{2}\left({ }_{a} D_{x}^{\gamma}[h](x)-{ }_{x} D_{b}^{\gamma}[h](x)\right), \quad x \in(a, b) .
$$

Similarly, the left, right and Riesz-Caputo fractional-order derivatives are defined by

$$
\begin{aligned}
& { }_{a}^{C} D_{x}^{\gamma}[h](x)=\frac{1}{\Gamma(1-\gamma)} \int_{a}^{x} \frac{h^{\prime}(\tau) d \tau}{(x-\tau)^{\gamma}}, \quad x \in(a, b] \\
& { }_{x}^{C} D_{b}^{\gamma}[h](x)=-\frac{1}{\Gamma(1-\gamma)} \int_{x}^{b} \frac{h^{\prime}(\tau) d \tau}{(\tau-x)^{\gamma}}, \quad x \in[a, b) \\
& { }_{a}^{C} D_{b}^{\gamma}[h](x)=\frac{1}{2}\left({ }_{a}^{C} D_{x}^{\gamma}[h](x)-{ }_{x}^{C} D_{b}^{\gamma}[h](x)\right), \quad x \in(a, b)
\end{aligned}
$$

where $h^{\prime}(x)$ denotes the first-order derivative of the function $h$ with respect to $x$. In what follows, we consider only the Riesz-RL and the Riesz-Caputo fractional-order derivatives. Note that when $h$ is continuously differentiable and its first-order derivative is integrable, the Riesz-RL and the Riesz-Caputo fractional-order derivatives are equivalent under the homogeneous boundary condition $[35,38]$. Due to the equivalency, both the fractional-order derivatives are uniformly denoted by $D^{\gamma}$. Moreover, since there is a singularity at the endpoint, we set $h(a)=h(b)=0$ for the fractional-order derivative.

Based on the definitions of the fractional-order derivatives, referring to [28] we give the definition of the fractional-order total variation. Let $C_{0}^{\infty}(\Omega)$ denote the space of infinitely continuously differentiable functions with compact support in $\Omega \subset \mathbb{R}^{2}$. The fractional-order total variation of a function $u$ is defined as

$$
\int_{\Omega}\left|D^{\gamma} u\right|:=\sup \left\{\int_{\Omega}-u d i v^{\gamma} \varphi d x \mid \varphi \in \mathcal{K}\right\}
$$

where $\mathcal{K}$ is the space of special test functions, which is formulated as

$$
\mathcal{K}:=\left\{\phi \in\left(C_{0}^{\infty}(\Omega)\right)^{2},\|\phi\|_{\infty} \leqslant 1\right\}
$$

Furthermore, $\operatorname{div}^{\gamma} \varphi=D_{x}^{\gamma} \varphi_{1}+D_{y}^{\gamma} \varphi_{2}$, where $D_{x}^{\gamma} \varphi_{1}$ and $D_{y}^{\gamma} \varphi_{2}$ denote the fractional-order derivatives along the $x$ - and $y$-direction, respectively. Then, the space of functions with $\gamma$-bounded variation $(\gamma-\mathrm{BV})$ in $\Omega$ is defined as follows,

$$
B V^{\gamma}(\Omega):=\left\{u \in L^{1}(\Omega)\left|\int_{\Omega}\right| D^{\gamma} u \mid<+\infty\right\} .
$$

With the $\gamma$-BV norm $\|u\|_{B V^{\gamma}}=\|u\|_{L^{1}}+\int_{\Omega}\left|D^{\gamma} u\right|$, the space $B V^{\gamma}(\Omega)$ is a Banach space [28].

At the end of this section, we give the definitions of the fractional-order derivatives under the discrete settings. For the sake of simplicity, we keep the same notations as in the continuous settings. Assume that $u \in \mathbb{R}^{n \times n}$ and satisfies Dirichlet boundary condition. According to [39,40], the discrete fractional-order derivatives at the point $(i, j)$ with the order $\gamma$ along the $x$ - and $y$ direction are respectively defined as

$$
\begin{aligned}
& D_{x}^{\gamma} u_{i, j}=\frac{1}{2}\left(\sum_{k=0}^{i+1} c_{k}^{\gamma} u_{i-k+1, j}+\sum_{k=0}^{n-i+2} c_{k}^{\gamma} u_{i+k-1, j}\right), \quad i, j=1, \cdots, n \\
& D_{y}^{\gamma} u_{i, j}=\frac{1}{2}\left(\sum_{k=0}^{j+1} c_{k}^{\gamma} u_{i, j-k+1}+\sum_{k=0}^{n-j+2} c_{k}^{\gamma} u_{i, j+k-1}\right), \quad i, j=1, \cdots, n
\end{aligned}
$$


where $c_{k}^{\gamma}=(-1)^{k}\left(\begin{array}{l}\gamma \\ k\end{array}\right)$ for $k=0,1, \cdots, n+1$. Numerically, we calculate the coefficients $c_{k}^{\gamma}$ through the following recursive relation

$$
c_{0}^{\gamma}=1, \quad c_{k}^{\gamma}=\left(1-\frac{1+\gamma}{k}\right) c_{k-1}^{\gamma}, \quad k=1,2, \cdots .
$$

Let $c=c_{0}^{\gamma}+c_{2}^{\gamma}$, the discrete fractional-order gradient of $u$ can be written as

$$
D^{\gamma} u=\left(\begin{array}{l}
D_{x}^{\gamma} u \\
D_{y}^{\gamma} u
\end{array}\right)=\left(\begin{array}{l}
M u \\
u M
\end{array}\right) \in \mathbb{R}^{2 n \times n}
$$

where

$$
M=\left(\begin{array}{ccccc}
2 c_{1}^{\gamma} & c & c_{3}^{\gamma} & \cdots & c_{n}^{\gamma} \\
c & 2 c_{1}^{\gamma} & \ddots & \ddots & \vdots \\
c_{3}^{\gamma} & \ddots & \ddots & \ddots & c_{3}^{\gamma} \\
\vdots & \ddots & \ddots & 2 c_{1}^{\gamma} & c \\
c_{n}^{\gamma} & \cdots & c_{3}^{\gamma} & c & 2 c_{1}^{\gamma}
\end{array}\right) \in \mathbb{R}^{n \times n} .
$$

Based on the symmetry of the matrix $M$ and the definition of the inner production on the matrix, i.e., $\langle A, B\rangle=\operatorname{tr}\left(A^{\top} B\right)=(\text { vec } A)^{\top}$ vec $B$ for all $A, B \in \mathbb{R}^{n \times n}$ where vec $A$ and vec $B$ are vectors by stacking all the columns of the matrix $A$ and $B$ respectively, we have

$$
\begin{aligned}
& \left\langle M u, v^{1}\right\rangle=\operatorname{tr}\left(u^{\top}\left(M v^{1}\right)\right)=\left\langle u, M v^{1}\right\rangle, \\
& \left\langle u M, v^{2}\right\rangle=\left(\operatorname{vec}\left(u M^{\top}\right)\right)^{\top} \operatorname{vec} v^{2}=(\operatorname{vec} u)^{\top} \operatorname{vec}\left(v^{2} M\right)=\left\langle u, v^{2} M\right\rangle,
\end{aligned}
$$

for any $v^{1}, v^{2} \in \mathbb{R}^{n \times n}$. Further, we obtain

$$
\left\langle D^{\gamma} u, v\right\rangle=\left\langle M u, v^{1}\right\rangle+\left\langle u M, v^{2}\right\rangle=\left\langle u, M v^{1}+v^{2} M\right\rangle, \text { for any } v=\left(\begin{array}{l}
v^{1} \\
v^{2}
\end{array}\right) \in \mathbb{R}^{2 n \times n} .
$$

Hence, we define the adjoint discrete fractional-order operator as $\left(D^{\gamma}\right)^{\top} v=M v^{1}+v^{2} M$.

\section{The Proposed Variational Models}

In [28], it has been shown that the fractional-order gradient can well represent features in images. In order to fuse the source images, we assume that the fractional-order gradient information of the fused image matches with the target fractional-order gradient feature, which is obtained by integrating the source images. Moreover, the fused image should be close to a pre-defined image $u_{0}$. To reduce the influence of the noise from the source images, we also incorporate TV regularization, which was proposed in [41] for image denoising, into our model. According to all the requirements, by using $L^{1}$ norm to measure the difference between $D^{\gamma} u$ and the target feature $w$, a new TV-based model is proposed for simultaneously fusing and denoising as follows

$$
\min _{u \in B V^{\gamma}(\Omega) \cap L^{2}(\Omega)} \int_{\Omega}|D u|+\alpha \int_{\Omega}\left|D^{\gamma} u-w\right| d x+\frac{\beta}{2} \int_{\Omega}\left|u-u_{0}\right|^{2} d x,
$$

where the positive regularization parameters $\alpha$ and $\beta$ control the balance among the three terms in the model, $w$ denotes the target fractional-order gradient feature, and $u_{0}$ is a pre-defined image. Further, the first term is the TV regularization, which is defined as

$$
\int_{\Omega}|D u|:=\sup \left\{\int_{\Omega} u \operatorname{div} \varphi d x \mid \varphi \in\left(C_{0}^{\infty}(\Omega)\right)^{2},\|\varphi\|_{\infty} \leqslant 1\right\} .
$$


Similarly, we propose another variational model by using $L^{2}$ norm to measure the difference between $D^{\gamma} u$ and $w$ :

$$
\min _{u \in B V^{\gamma}(\Omega) \cap L^{2}(\Omega)} E(u):=\int_{\Omega}|D u| d x+\frac{\alpha}{2} \int_{\Omega}\left|D^{\gamma} u-w\right|^{2} d x+\frac{\beta}{2} \int_{\Omega}\left|u-u_{0}\right|^{2} d x .
$$

We are able to prove the existence and uniqueness to the solution for the TVL2 model.

Theorem 3.1. Assume that $u_{0} \in B V^{\gamma}(\Omega) \cap L^{2}(\Omega)$ and $w$ has a finite vector-valued Radon measure, then the minimization problem in (TVL2) has a unique minimizer $u^{*} \in B V^{\gamma}(\Omega) \cap L^{2}(\Omega)$.

Proof. In order to prove the existence and uniqueness of a solution, we first set $\left\{u^{k}\right\} \subset B V^{\gamma}(\Omega) \cap$ $L^{2}(\Omega)$ to be a minimizing sequence of (TVL2). Since $E(0)$ is finite and $E(u)$ is positive, the minimum of the energy function $E(u)$ is also finite. In other words, there exists a positive constant $C$ such that

$$
E\left(u^{k}\right)=\int_{\Omega}\left|D u^{k}\right|+\frac{\alpha}{2} \int_{\Omega}\left|D^{\gamma} u^{k}-w\right|^{2} d x+\frac{\beta}{2} \int_{\Omega}\left|u^{k}-u_{0}\right|^{2} d x \leqslant C .
$$

Since $w$ has the finite vector-valued Radon measure, we have that

$$
\int_{\Omega}|w|:=\sup \left\{\int_{\Omega} w \cdot \varphi d x \mid \varphi \in \mathcal{K}\right\}<+\infty
$$

Due to the fact that $\int_{\Omega}\left|D^{\gamma} u^{k}-w\right| d x:=\sup \left\{\int_{\Omega}\left(-u^{k} d i v^{\gamma} \varphi+w \cdot \varphi\right) d x \mid \varphi \in \mathcal{K}\right\}$, then we obtain the following inequality,

$$
\int_{\Omega}\left|D^{\gamma} u^{k}\right| \leqslant \int_{\Omega}\left|D^{\gamma} u^{k}-w\right| d x+\int_{\Omega}|w| \leqslant C
$$

Since $E\left(u^{k}\right) \leqslant C$, we have

$$
\int_{\Omega}\left|u^{k}-u_{0}\right|^{2} d x \leqslant C
$$

Further, according to $u_{0} \in L^{2}(\Omega)$ and $L^{2}(\Omega) \subset L^{1}(\Omega)$ we deduce that

$$
\int_{\Omega}\left|u^{k}\right|^{2} d x \leqslant C, \quad \int_{\Omega}\left|u^{k}\right| d x \leqslant C .
$$

Combining with (4) and (6), we have that $\left\{u^{k}\right\}$ is bounded in $B V^{\gamma}(\Omega) \cap L^{2}(\Omega)$. Based on the weak* topology of $B V^{\gamma}(\Omega)$ [28] and the reflexivity of $L^{2}(\Omega)$, there exists a subsequence that converges to $u^{*} \in B V^{\gamma}(\Omega) \cap L^{2}(\Omega)$ such that

$$
u^{k} \stackrel{B V^{\gamma}-w^{*}}{\longrightarrow} u^{*} \quad \text { and } \quad u^{k} \stackrel{L^{2}(\Omega)}{\longrightarrow} u^{*} .
$$

Due to the lower semi-continuity of TV and the fractional-order total variation [28], we have

$$
\begin{aligned}
& \int_{\Omega}\left|D u^{*}\right| \leqslant \liminf _{k \rightarrow \infty} \int_{\Omega}\left|D u^{k}\right|, \\
& \int_{\Omega}\left|D^{\gamma} u^{*}-w\right|^{2} d x \leqslant \liminf _{k \rightarrow \infty} \int_{\Omega}\left|D^{\gamma} u^{k}-w\right|^{2} d x .
\end{aligned}
$$

Therefore, by Fatou's lemma, we can conclude that $E\left(u^{*}\right) \leqslant \liminf _{k \rightarrow \infty} E\left(u^{k}\right)$ and $u^{*}$ is a minimizer of (TVL2). Moreover, since the energy function $E(u)$ is obviously strictly convex, the minimizer $u^{*}$ is unique. 
Remark 1. In both TVL1 and TVL2 models, the second terms are used to incorporate important salient features from the source images according to the fractional-order derivatives. Referring to the selection criterion introduced in $[23,42,43]$, here we propose a relatively simple way to obtain the target fractional-order feature $w$ from the source images. We assume that two source images are $f_{1}$ and $f_{2}$ in $\mathbb{R}^{n \times n}$, and compute the fractional-order gradient $D^{\gamma} f_{i}=\left(\begin{array}{c}M f_{i} \\ f_{i} M\end{array}\right)$ for $i=1,2$. Then, the target fractional-order feature $w$ is formulated as

$$
w=s D^{\gamma} f_{1}+(1-s) D^{\gamma} f_{2},
$$

where $s$ denotes a binary mask for fusing the salient features from the noisy source images. By smoothing and thresholding, the binary mask is defined as

$$
s_{i, j}=\left\{\begin{array}{ll}
1, & \text { if }(K * \tilde{s})_{i, j}>0.5 \\
0, & \text { otherwise, }
\end{array} \quad \text { with } \quad \tilde{s}_{i, j}= \begin{cases}1, & \text { if }\left(K *\left(D^{\gamma} f_{1}\right)^{2}\right)_{i, j}>\left(K *\left(D^{\gamma} f_{2}\right)^{2}\right)_{i, j} \\
0, & \text { otherwise, }\end{cases}\right.
$$

where $*$ represents the convolution operation and $K \in \mathbb{R}^{\omega \times \omega}$ is a mean kernel, i.e., all elements in $K$ equal $\frac{1}{\omega^{2}}$. Note that when there are more than two source images, we utilize the recursive method and only incorporate two source images each time.

Remark 2. In our models, the data-fitting term $\int_{\Omega}\left|u-u_{0}\right|^{2} d x$ depends on a pre-defined image, and the quality of $u_{0}$ has direct effect on the capability of our methods for fusing images. In order to preserve the image contrast, we combine the average contrast $(A C)$ introduced in [23] with the same selection criterion as in Remark 1. Specifically, if $A C\left(f_{1}\right)-A C\left(f_{2}\right)>0.5$, then $u_{0}=s f_{1}+(1-s) f_{2}$ where $s$ satisfies the equation (7); otherwise, $u_{0}$ equals to the average of the source images.

\section{Numerical Algorithms}

Since the minimization problems in the TVL1 and TVL2 models are convex, many numerical methods can be used to solve them, e.g. the gradient descent method [41], the primal-dual method [44,45], the split Bregman method [25] and the alternating minimization method [46-48]. In this section, we apply the ADMM algorithm [29-31,49,50] to solve them due to the fast convergence in convex optimization. Hereafter, we consider the proposed models in the discrete setting, and for the sake of simplicity we keep the same notations.

First, we focus on the TVL2 model, and its discrete form can be written as

$$
\min _{u \in \mathbb{R}^{n \times n}}\|D u\|_{1}+\frac{\alpha}{2}\left\|D^{\gamma} u-w\right\|_{F}^{2}+\frac{\beta}{2}\left\|u-u_{0}\right\|_{F}^{2},
$$

where $u \in \mathbb{R}^{n \times n}$ denotes a gray-scale image and $\|\cdot\|_{F}$ represents the Frobenius norm. The gradient of $u$ under the zero boundary condition is formulated as

$$
D u=\left(\begin{array}{c}
N u \\
u N^{\top}
\end{array}\right) \in \mathbb{R}^{2 n \times n},
$$

where

$$
N=\left(\begin{array}{cccc}
1 & & & \\
-1 & 1 & & \\
& \ddots & \ddots & \\
& & -1 & 1
\end{array}\right) \in \mathbb{R}^{n \times n}
$$


Then, we have $\|D u\|_{1}=\sum_{i=1}^{n} \sum_{j=1}^{n} \sqrt{(N u)_{i, j}^{2}+\left(u N^{\top}\right)_{i, j}^{2}}$.

In order to apply the ADMM algorithm, we introduce an auxiliary variable $v \in \mathbb{R}^{2 n \times n}$ and rewrite (8) as the following constraint convex optimization problem

$$
\begin{gathered}
\min _{u \in \mathbb{R}^{n \times n}, v \in \mathbb{R}^{2 n \times n}}\|v\|_{1}+\frac{\alpha}{2}\left\|D^{\gamma} u-w\right\|_{F}^{2}+\frac{\beta}{2}\left\|u-u_{0}\right\|_{F}^{2}, \\
\text { s.t. } v=D u .
\end{gathered}
$$

Let $p \in \mathbb{R}^{2 n \times n}$ be the Lagrangian multiplier for the linear constraint $v=D u$, and we give the corresponding augmented Lagrangian function,

$$
\mathcal{L}(u, v, p)=\|v\|_{1}+\frac{\alpha}{2}\left\|D^{\gamma} u-w\right\|_{F}^{2}+\frac{\beta}{2}\left\|u-u_{0}\right\|_{F}^{2}+\langle p, D u-v\rangle+\frac{\delta}{2}\|D u-v\|_{F}^{2}
$$

where $\delta>0$ is the penalty parameter. Under the framework of ADMM algorithm, the subproblems with respect to $u$ and $v$ are solved alternately.

The solution of the $v$-subproblem has a closed-form solution, and can be obtained by applying the soft shrinkage operator [46], i.e.,

$$
\begin{aligned}
v^{k+1} & =\underset{v}{\arg \min }\|v\|_{1}+\frac{\delta}{2}\left\|D u^{k}-v+\frac{p^{k}}{\delta}\right\|_{F}^{2} \\
& =\operatorname{shrink}\left(D u^{k}+\frac{p^{k}}{\delta}, \frac{1}{\delta}\right)
\end{aligned}
$$

where $\operatorname{shrink}(x, \rho)=\max \left(\|x\|_{F}-\rho, 0\right) \cdot \frac{x}{\|x\|_{F}}$, obeying the convention $0 \cdot \frac{0}{0}=0$. For the $u$-subproblem, the minimization problem is

$$
u^{k+1}=\underset{u}{\arg \min } \frac{\alpha}{2}\left\|D^{\gamma} u-w\right\|_{F}^{2}+\frac{\beta}{2}\left\|u-u_{0}\right\|_{F}^{2}+\left\langle p^{k}, D u-v^{k+1}\right\rangle+\frac{\delta}{2}\left\|D u-v^{k+1}\right\|_{F}^{2} .
$$

Based on the first-order optimality condition [51], the solution should satisfy the following linear equation:

$$
\left(\alpha\left(D^{\gamma}\right)^{\top} D^{\gamma}+\delta D^{\top} D+\beta I\right) u=\alpha\left(D^{\gamma}\right)^{\top} w+D^{\top}\left(\delta v^{k+1}-p^{k}\right)+\beta u_{0} .
$$

According to the definitions of the operators $D$ and $D^{\gamma}$, we obtain the following Sylvester-like equation

$$
\left(\alpha M^{2}+\delta N^{\top} N+\beta I\right) u+u\left(\alpha M^{2}+\delta N^{\top} N\right)=\alpha\left(D^{\gamma}\right)^{\top} w+D^{\top}\left(\delta v^{k+1}-p^{k}\right)+\beta u_{0},
$$

which can be solved by the conjugate gradient (CG) method [52]. The overall algorithm is described in Algorithm 1.

Finally, motivated by the works in $[53,54]$, we give the following theorem to show the convergence of Algorithm 1.

Theorem 4.1. For the fixed parameter $\delta>0$ and $\tau \in\left(0, \frac{\sqrt{5}+1}{2}\right)$, the ADMM algorithm for solving the TVL2 model (9) converges.

This theorem can be considered as a special case of the convergence theorems in [53,54]. Note that although the $u$-subproblem is approximatively solved by the conjugate gradient (CG) method, Algorithm 1 is empirically convergent. 


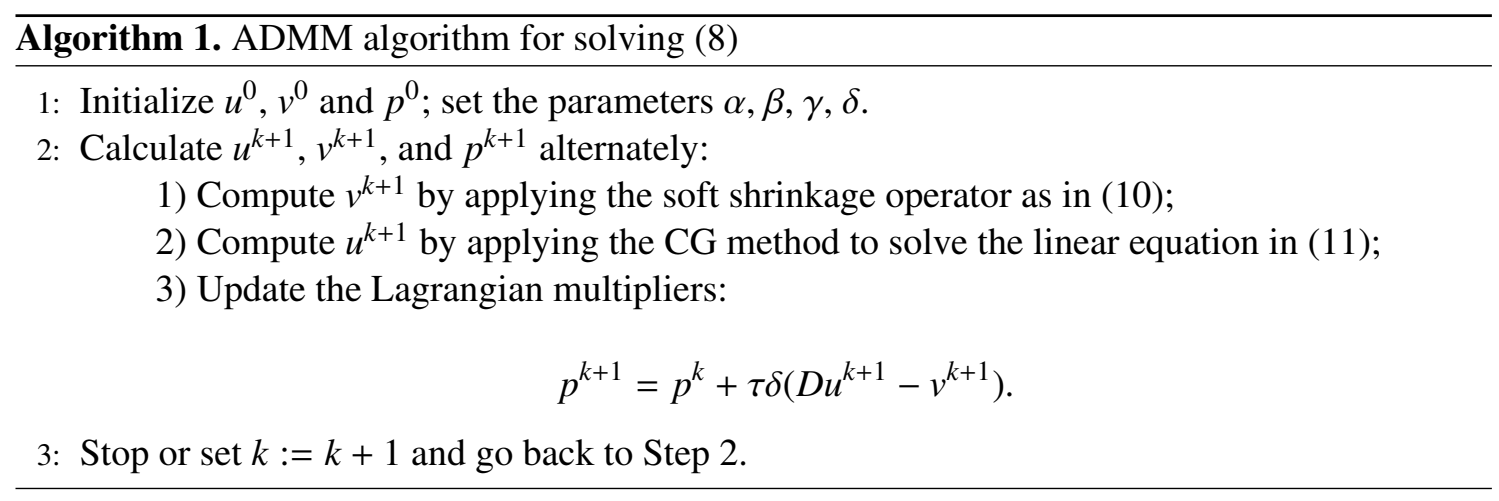

In order to apply the ADMM algorithm to solve the TVL1 model, we give the corresponding discrete formulation:

$$
\min _{u \in \mathbb{R}^{n \times n}}\|D u\|_{1}+\alpha\left\|D^{\gamma} u-w\right\|_{1}+\frac{\beta}{2}\left\|u-u_{0}\right\|_{F}^{2}
$$

we introduce two variables $v \in \mathbb{R}^{2 n \times n}$ and $t \in \mathbb{R}^{2 n \times n}$ and rewrite (12) as the following constrained minimization problem

$$
\begin{gathered}
\min _{u, v, t}\|v\|_{1}+\alpha\|t\|_{1}+\frac{\beta}{2}\left\|u-u_{0}\right\|_{F}^{2}, \\
\text { s.t. } v=D u, t=D^{\gamma} u-w .
\end{gathered}
$$

Then, the corresponding augmented Lagrangian function of (13) is

$$
\begin{aligned}
\mathcal{L}\left(u, v, t, p_{1}, p_{2}\right) & =\|v\|_{1}+\alpha\|t\|_{1}+\frac{\beta}{2}\left\|u-u_{0}\right\|_{F}^{2}+\left\langle p_{1}, D u-v\right\rangle+\frac{\delta}{2}\|D u-v\|_{F}^{2} \\
& +\left\langle p_{2}, D^{\gamma} u-w-t\right\rangle+\frac{\delta}{2}\left\|D^{\gamma} u-w-t\right\|_{F}^{2}
\end{aligned}
$$

where $p_{1} \in \mathbb{R}^{2 n \times n}$ and $p_{2} \in \mathbb{R}^{2 n \times n}$ denote the Lagrangian multipliers, $\delta$ is the positive penalty parameter. Similar to Algorithm 1, we solve the subproblems with respect to $u, v$ and $t$ alternately, and the whole algorithm is shown in Algorithm 2.

\section{Numerical Experiments}

In this section, we present several numerical experiments to illustrate the superior performance of the proposed methods for fusing the noisy source images. All numerical experiments are performed under Windows 8 and Matlab (R2015b) running on a desktop with 3.40GHz Intel Core i3-2130 central processing unit (CPU) and 4G random-access memory (RAM) memory. In order to show the potentiality of the proposed methods, we compare with some existing popular algorithms: the WTV method from [20] and the FSG method from [23]. Since the FSG method cannot deal with noisy source images, we apply the L2-TV method introduced in [41] to denoise the source images first, and then use the FSG method to fuse them.

In real applications, it is not easy to quantitatively measure the quality of the fused results due to the absence of the true original image. According to [55], for evaluating the quality of the fused results, we consider two objective fusion metrics: mutual information metric and phase congruency metric. The mutual information based fusion metric $[56,57]$ is defined by

$$
Q_{M I}=M I\left(u^{*}, f_{1}\right)+M I\left(u^{*}, f_{2}\right)
$$


Algorithm 2. ADMM algorithm for solving (12)

1: Initialize $u^{0}, v^{0}, t^{0}, p_{1}^{0}$ and $p_{2}^{0}$; set the parameters $\alpha, \beta, \gamma, \delta$.

2: Calculate $u^{k+1}, v^{k+1}, t^{k+1}, p_{1}^{k+1}$ and $p_{2}^{k+1}$ alternately:

1) Compute $v^{k+1}$ and $t^{k+1}$ by applying the soft shrinkage operator:

$$
\begin{aligned}
v^{k+1} & =\operatorname{shrink}\left(D u^{k}+\frac{p_{1}^{k}}{\delta}, \frac{1}{\delta}\right) \\
t^{k+1} & =\operatorname{shrink}\left(D^{\gamma} u^{k}-w+\frac{p_{2}^{k}}{\delta}, \frac{\alpha}{\delta}\right)
\end{aligned}
$$

2) Compute $u^{k+1}$ by applying the CG method to solve the following linear equation:

$$
\left(\delta\left(D^{\gamma}\right)^{\top} D^{\gamma}+\delta D^{\top} D+\beta I\right) u=\delta\left(D^{\gamma}\right)^{\top}\left(w+t^{k+1}-p_{2}^{k}\right)+D^{\top}\left(\delta v^{k+1}-p_{1}^{k}\right)+\beta u_{0} .
$$

3) Update the Lagrangian multipliers:

$$
\begin{aligned}
& p_{1}^{k+1}=p_{1}^{k}+\tau \delta\left(D u^{k+1}-v^{k+1}\right) \\
& p_{2}^{k+1}=p_{2}^{k}+\tau \delta\left(D^{\gamma} u^{k+1}-w-t^{k+1}\right)
\end{aligned}
$$

3: Stop or set $k:=k+1$ and go back to Step 2 .

where $u^{*}$ is the fused image, $f_{1}$ and $f_{2}$ are the source images. The mutual information operation $M I(x, y)$ can be expressed as

$$
M I(x, y)=H(x)+H(y)-H(x, y)
$$

with

$$
\begin{aligned}
H(x) & =-\sum_{x} p(x) \log _{2} p(x) \\
H(x, y) & =-\sum_{x, y} p(x, y) \log _{2} p(x, y),
\end{aligned}
$$

where $p(x)$ is the marginal probability distribution function and $p(x, y)$ is the joint probability distribution function. The higher $Q_{M I}$ values implies the better fused results. By combining with the principal (maximum and minimum) moments of the image phase congruency, another fusion metric $[58,59]$ is defined as follows,

$$
Q_{P}=P_{p} P_{M} P_{m}
$$

where $p$ is the phase congruency, $M$ is the maximum moment, $m$ is the minimum moment, $P_{p}=$ $\max \left\{C_{f_{1}, u^{*}}^{p}, C_{f_{2}, u^{*}}^{p}, C_{s, u^{*}}^{p}\right\}, P_{M}=\max \left\{C_{f_{1}, u^{*}}^{M}, C_{f_{2}, u^{*}}^{M}, C_{s, u^{*}}^{M}\right\}$, and $P_{m}=\max \left\{C_{f_{1}, u^{*}}^{m}, C_{f_{2}, u^{*}}^{m}, C_{s, u^{*}}^{m}\right\}$. Note that $s$ is the maximum-select map. The correlation coefficient between two sets $x$ and $y$ is given by

$$
C_{x, y}^{k}=\frac{\sigma_{x y}^{k}+C}{\sigma_{x}^{k} \sigma_{y}^{k}+C},
$$

where $k \in\{p, M, m\}, \sigma_{x}$ and $\sigma_{y}$ are their respective standard variances, $\sigma_{x y}$ are the covariance of $x$ and $y$, and $C>0$ is a constant. 
Table 1. The values of $Q_{M I}$ and $Q_{P}$ by using different methods for the test image "Cameraman".

\begin{tabular}{ccccccccc}
\hline & \multicolumn{4}{c}{$Q_{M I}$} & \multicolumn{4}{c}{$Q_{P}$} \\
\cline { 2 - 9 } Noise & FSG & WTV & TVL1 & TVL2 & FSG & WTV & TVL1 & TVL2 \\
\hline 15 & 5.8297 & 5.8548 & 6.0030 & 6.0384 & 0.5053 & 0.4955 & 0.5232 & 0.5225 \\
20 & 5.5549 & 5.6269 & 5.7807 & 5.8035 & 0.4297 & 0.4353 & 0.4463 & 0.4519 \\
25 & 5.2479 & 5.4410 & 5.6076 & 5.6160 & 0.3702 & 0.3935 & 0.4012 & 0.4050 \\
30 & 4.9419 & 5.2760 & 5.4735 & 5.4823 & 0.3194 & 0.3583 & 0.3660 & 0.3647 \\
\hline
\end{tabular}

For the fair comparisons, we set the same maximum iteration number as 100 in the FSG, TVL1 and TVL2 methods. In addition, the stopping criterion is that the relative difference between the successive iterates should be small enough, which is measured by

$$
\frac{\left\|u^{k+1}-u^{k}\right\|_{2}}{\left\|u^{k}\right\|_{2}} \leqslant 10^{-3} .
$$

In Algorithm 1 and 2, the parameter $\tau$ controls the convergence speed. According to the convergence theorem, we set $\tau=1.618$ and the penalty parameter $\delta$ is set in the interval $[0.1,1]$. The maximum iteration number for the CG method equals 5 as solving the $u$-subproblem. In the TVL1 and TVL2 models, the regularization parameters $\alpha$ and $\beta$ control the trade-off among the smoothness from the TV regularization, the fitting to the target feature, and the fitting to the predefined image. Here, we manually tune them for obtaining the best fused results. In addition, the fractional-order parameter $\gamma$ lies in the interval $[1,2]$. We also tune them by hands for getting the highest values of $Q_{M I}$.

\subsection{Experiment on Simulated Images}

In this experiment, we use the test image "Cameraman", which is blurred in the left part and right part respectively with a Gaussian blurring kernel (standard deviation $\sigma=2$, window size $5 \times 5$ ). Then the Gaussian noise is added to the blurring images, where the noise level $\sigma \in\{15,20,25,30\}$, respectively. We compare the fused results by using the FSG, WTV, TVL1 and TVL2 methods, and in Table 1 we list their $Q_{M I}$ and $Q_{P}$ values. It is obvious that TVL1 and TVL2 give the best fusion results with respect to $Q_{M I}$. In the most cases our methods outperform FSG and WTV with respect to $Q_{P}$ as well. Furthermore, TVL2 is always slightly better than TVL1.

Figure 1 shows the fused images by using different methods on the test image "Cameraman", where the noise level $\sigma=30$. We can clearly see that the artifacts from the denoising step propagate to fusion result in FSG, however WTV, TVL1 and TVL2 can avoid the influence of the noise in the source images. Unfortunately, the WTV method suffers from some undesired false edges. The TVL1 method products some artifacts in the background. The fused image by solving the TVL2 model is obviously better than the ones from the WTV and TVL1 models. The underline reasons that the proposed models outperform other variational models are given as follows. Firstly, the presence of the noise in fused images and the errors introduced during the denoising step would greatly affected the fused results, so FSG provides the worst fused images. Secondly, we use the TV regularization in the proposed models, and it can effectively remove the noise while preserve the sharp edges. Finally, we use the fractional-order derivative, which can effective represent image features, in the fusing fidelity term, so it can keep the smoothness in homogeneous regions with sharp image features. 


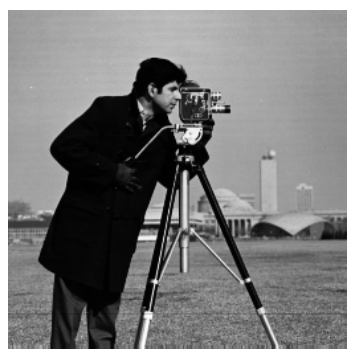

(a)

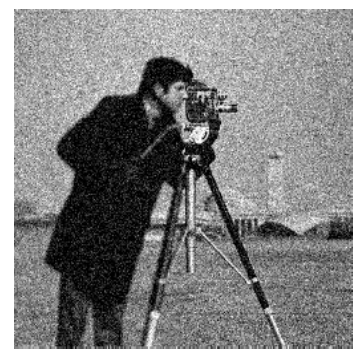

(b)

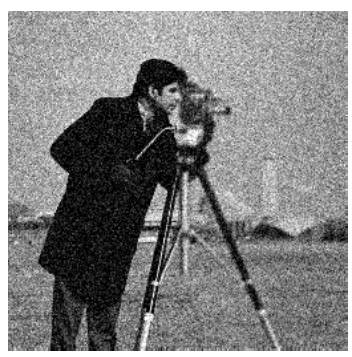

(c)

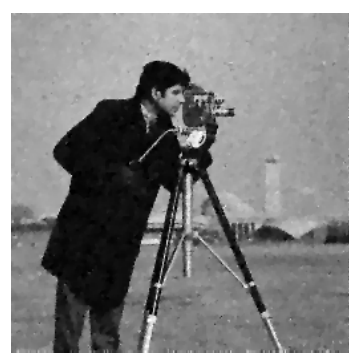

(d)

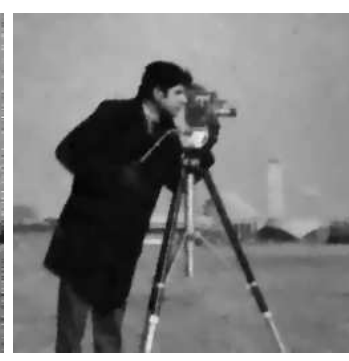

(e)

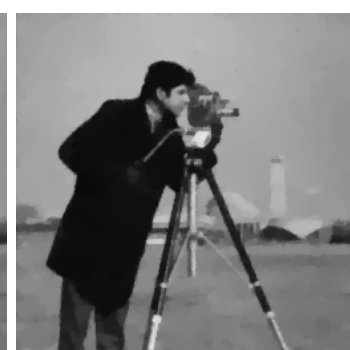

(f)

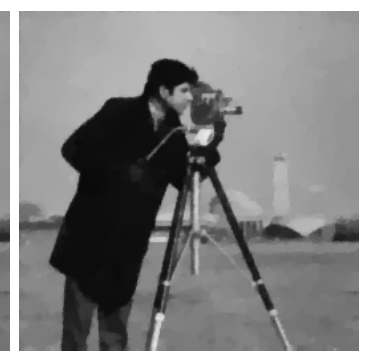

(g)

Figure 1. Comparison of different methods for fusing the noisy source images, the noise level $\sigma=30$. (a) The original image; (b) and (c) two source images by blurring the left part and right part, respectively; (d) FSG [23]; (e) WTV [20]; (f) TVL1; (g) TVL2.

In order to further illustrate the performance of the four methods, in Figure 2 we show the corresponding edge maps of the fused images by using the canny edge detector. From the edge maps, we can see that the TVL2 method outperforms the other three methods. In the edge map from FSG, there exists some false edges due to error propagation. Comparing with the edge maps from WTV and TVL1, the one from TVL2 has no wrong edges in the region of the sky and the black coat.

\subsection{Experiments on Real Images}

In this section, we show some numerical results on fusing real images. Figure 3 shows the real images in the test, including three pairs of multi-focus images and five pairs of multi-modal images, which can be found in the website http://www.quxiaobo.org/index.html. In order to show the capability on dealing with noisy source images, we add white Gaussian noise with the standard variance $\sigma \in\{15,20,25,30\}$, respectively, on the real test images. Table 2 lists the values of $Q_{M I}$ and $Q_{P}$ by using four different methods on the real multi-focus images. Obviously TVL2 provides the highest $Q_{M I}$ values, and also in the most cases gives the highest $Q_{P}$ values. Especially, when the noise level is high, the results from TVL2 can show the notable improvement.

Figure 4 and 6 show the fused images by applying four different methods, where the noise level are $\sigma=20$ and 30, respectively. Since FSG can only fuse the noise-free images, its results shown in (c) still retains some noise or artifacts from denoising. The WTV method is able to remove the noise, but also its results are over-smoothing. Compared with FSG and WTV, the fused results from our methods are obviously better. Furthermore, the results from TVL2 preserve more textures than the ones from TVL1. In conclusion, the proposed TVL2 method can effectively remove the noise as well as fuse the salient features from the source images. In Figure 5 we show the zoomed parts of the fused images by using different methods. We can see that the textures of 


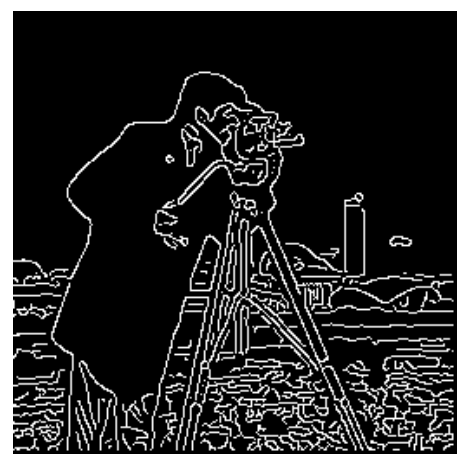

(a)

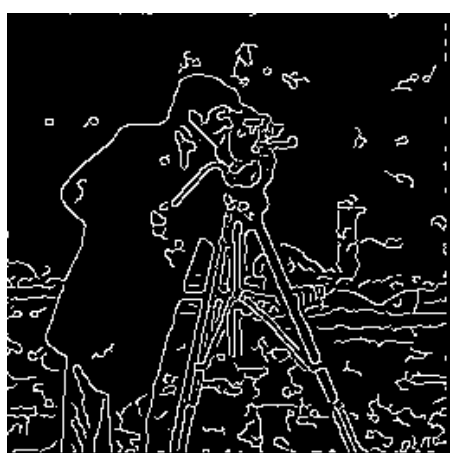

(b)

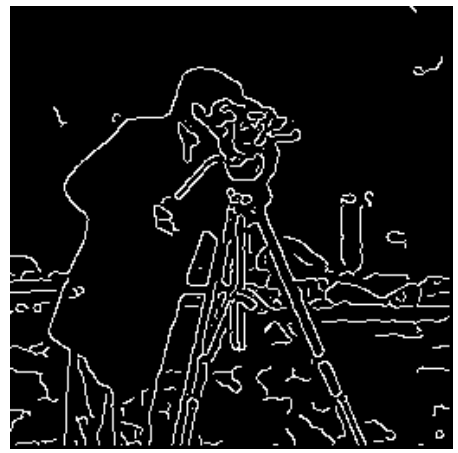

(c)

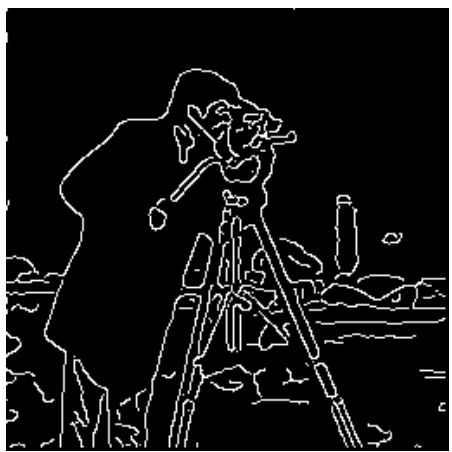

(d)

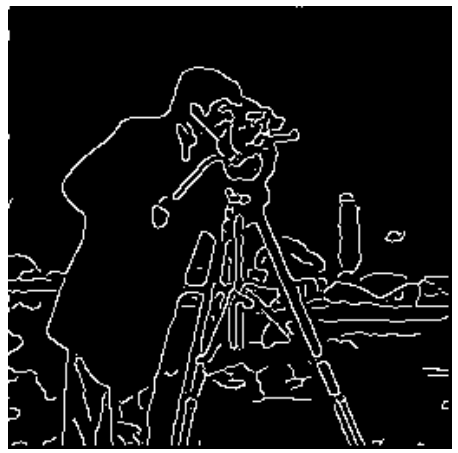

(e)

Figure 2. Edge maps of the fused images in Figure 1 by using the canny edge detector. (a) The original image; (b) FSG [23]; (c) WTV [20]; (d) TVL1; (f) TVL2.

the leaves in the results from TVL2 are clearer than from TVL1.

Next, we show the numerical results for fusing multi-modal images by using four different methods. Table 3 lists the $Q_{M I}$ and $Q_{P}$ values by applying four methods, where the noise level $\sigma=15,20,25,30$, respectively. In the most cases, the TVL1 and TVL2 methods give the higher values with respect to $Q_{M I}$ and $Q_{P}$ than the FSG and WTV methods. Moreover, the TVL2 method almost always outperforms the TVL1 method.

Figure 7 shows the comparison of different methods for fusing the noisy computed tomography (CT) image and the noisy magnetic resonance imaging (MRI) image, where the noise level $\sigma=20$ and 30, respectively. Figure 7(a) shows the CT images which well provide the detail structure of bones, Figure 7(b) shows the MRI images which mainly illuminate the detail structure of soft tissues. The results from FSG are still affected by the noise and denoising artifacts, and the other three methods are effective on dealing with noisy source images. But the WTV method provides darker images and reduces the image contrast. The TVL1 and the TVL2 methods perform obviously better. From the zoomed parts shown in 8 we can see that there are some undesired artifacts in the results from TVL1. However, TVL2 can fuse abundant information and salient features from the noisy source images.

Figure 9 and 10 are a comparison of different methods for fusing the noisy visible image and the noisy infrared image. The visible image captures many spatial details and background information, while the infrared image easily determines the position of thermal objects. The visible image is different from the infrared image, and the information in the visible image and the infrared image is complementary. Comparing with the results from FSG and WTV, in the results from the proposed TVL1 and TVL2 methods all the salient features are fairly clear. Furthermore, TVL2 


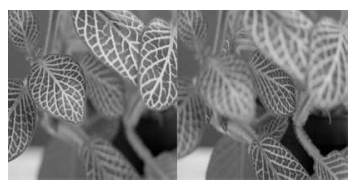

(a) Leaves $(204 \times 204)$

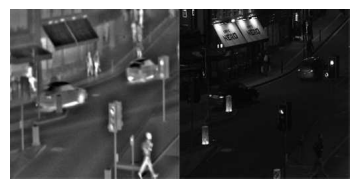

(e) Crossroad $(256 \times 256)$

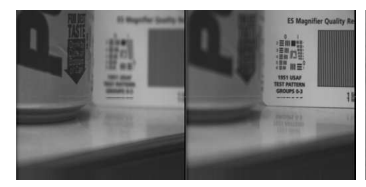

(b) Book $(256 \times 256)$

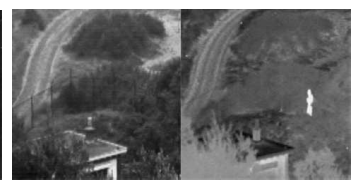

(f) Hill $(240 \times 240)$

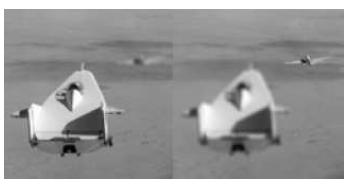

(c) Plane $(160 \times 160)$

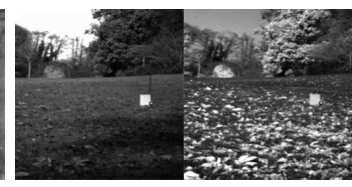

(g) Grass $(256 \times 256)$

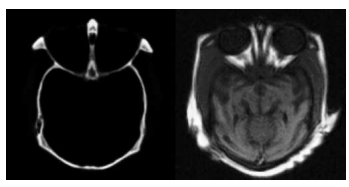

(d) Brain $(256 \times 256)$

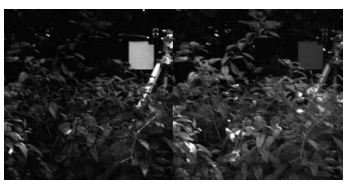

(h) Plant $(256 \times 256)$

Figure 3. Source images.

Table 2. The values of $Q_{M I}$ and $Q_{P}$ by using different methods for the real multi-focus images.

\begin{tabular}{cccccccccc}
\hline & & \multicolumn{9}{c}{$Q_{M I}$} & \multicolumn{4}{c}{$Q_{P}$} \\
\cline { 2 - 9 } Image & Noise & FSG & WTV & TVL1 & TVL2 & FSG & WTV & TVL1 & TVL2 \\
\hline \multirow{4}{*}{ Leaves } & 15 & 4.0423 & 3.9987 & 4.1412 & 4.1429 & 0.4240 & 0.3299 & 0.3649 & 0.3654 \\
& 20 & 3.9394 & 3.8466 & 4.0124 & 4.0286 & 0.3597 & 0.2762 & 0.2955 & 0.2970 \\
& 25 & 3.8310 & 3.7377 & 3.9049 & 3.9176 & 0.2864 & 0.2370 & 0.2436 & 0.2479 \\
& 30 & 3.7320 & 3.6350 & 3.8149 & 3.8234 & 0.2353 & 0.2086 & 0.2055 & 0.2134 \\
\hline \multirow{3}{*}{ Book } & 15 & 5.8238 & 6.0771 & 6.0888 & 6.1012 & 0.3321 & 0.3343 & 0.3337 & 0.3449 \\
& 20 & 5.6559 & 5.8697 & 5.9017 & 5.9269 & 0.2832 & 0.2952 & 0.2956 & 0.2956 \\
& 25 & 5.4851 & 5.6822 & 5.7374 & 5.7562 & 0.2317 & 0.2649 & 0.2692 & 0.2700 \\
& 30 & 5.3122 & 5.5135 & 5.6005 & 5.6284 & 0.1936 & 0.2415 & 0.2424 & 0.2475 \\
\hline \multirow{3}{*}{ Plane } & 15 & 5.3497 & 5.6256 & 5.6440 & 5.6492 & 0.3204 & 0.3722 & 0.3469 & 0.3502 \\
& 20 & 5.0658 & 5.3921 & 5.4187 & 5.4153 & 0.2680 & 0.3335 & 0.3149 & 0.3188 \\
& 25 & 4.7446 & 5.1903 & 5.2372 & 5.2373 & 0.2206 & 0.3022 & 0.2894 & 0.2913 \\
& 30 & 4.4491 & 5.0092 & 5.0695 & 5.0711 & 0.1828 & 0.2754 & 0.2555 & 0.2655 \\
\hline
\end{tabular}

provides better fused images without undesired artifacts.

\subsection{Influence of the model parameters}

In our methods, there are three parameters, $\alpha, \beta$ and $\gamma$, in the TVL1 and TVL2 models. The regularization parameters $\alpha$ and $\beta$ control the trade-off among the smoothness from the TV regularization, the fitting to the target feature, and the fitting to the pre-defined image. The parameter $\gamma$ in the fractional-order gradient decides the order of the derivative that is used for representing image features. In the previous experiments, we manually tune all these three parameters and pick up the one that gives the largest $Q_{M I}$ values. In this section, we test their influence on the fusion results.

Here, we use the source images "Leave" shown in Figure 3(a) as the test images, and the noise level is set as $\sigma=20$. Figure 11 shows the plot of the $Q_{M I}$ values of the fused results from our methods with varying $\alpha, \beta$ and $\gamma$, respectively. We can see that the curves from varying the regularization parameters $\alpha$ and $\beta$ have single peak. The reason is that $\alpha$ and $\beta$ control the fitting to the target image features and to the pre-defined images, respectively. By increasing these two parameters, the results changes from underfitting to overfitting. According to the range of $Q_{M I}$ values, we find that TVL2 is more sensitive to these two parameters. From Figure 11(c), we note 


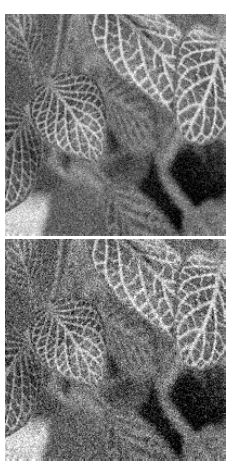

(a)

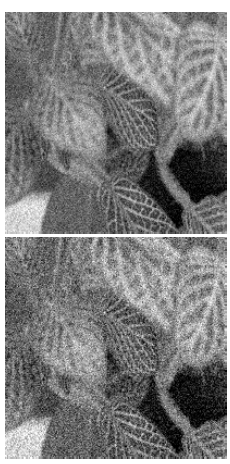

(b)

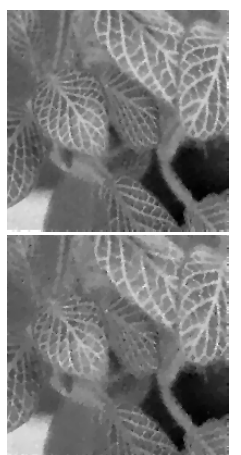

(c)

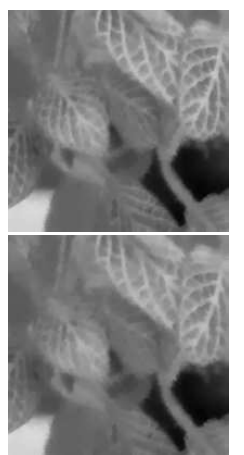

(d)

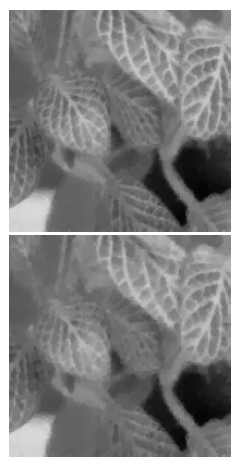

(e)

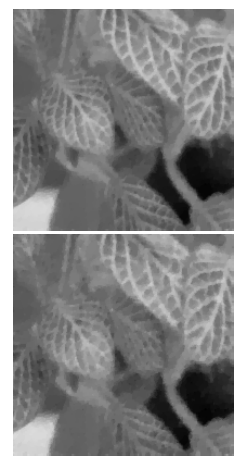

(f)

Figure 4. Comparison of different models for the images "Leaves", where the white Gaussian noise $\sigma=20$ (in the 1st row) and $\sigma=30$ (in the 2nd row), respectively. (a) and (b): two input source images; (c) FSG [23]; (d) WTV [20]; (e) TVL1; (f) TVL2.

that TVL2 is rather robust with respect to $\gamma$, and TVL1 prefers rather smaller $\gamma$ value.

\subsection{Comparison of the CPU times}

In Table 4, we list the CPU times (in seconds) consumed for solving the four different models under the same stopping criteria. In this test, the source images are corrupted by the additive Gaussian noise with the noise level $\sigma=20$. We find that the CPU times for all four methods are comparable. In the most cases, WTV is the fastest one, since it only takes the first order gradient information into account. Our methods need a bit more CPU time than WTV, but much less than FSG. The reason is that our methods require much less iterations until converging to satisfactory fused results than FSG. Furthermore, FSG requires some extra computations for pre-processing source images in order to remove noise, which is not counted here.

\section{Conclusion}

In this paper, we propose two novel variational models for image fusion. Due to the global features, we utilize the synthetical fractional-order derivative information from the source images as the prior for obtaining fused results. By incorporating the total variation (TV) regularization to remove the noise, the proposed models are able to fuse noisy source images. We apply the alternating direction method of multiplier (ADMM) for solving the optimization problems in the models. Numerical experiments show that our methods outperform the state-of-the-art methods in terms of visual and quantitative measures.

Although the proposed models give better fused results, it relies on manually tuning the regularization parameters in the models. An adaptive parameter selection method would be considered in the future.

\section{Acknowlegenments}

We would like to thank Fang Li from East China Normal University and Weiwei Wang from Xidian University for providing the software codes [23] and [20]. 

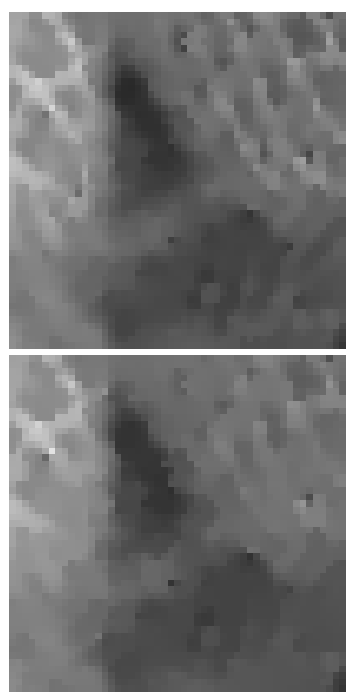

(a)
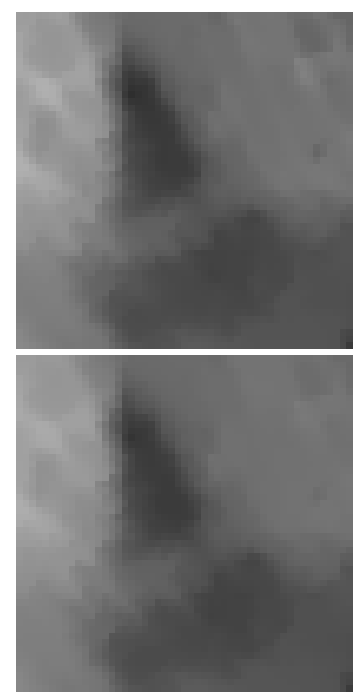

(b)

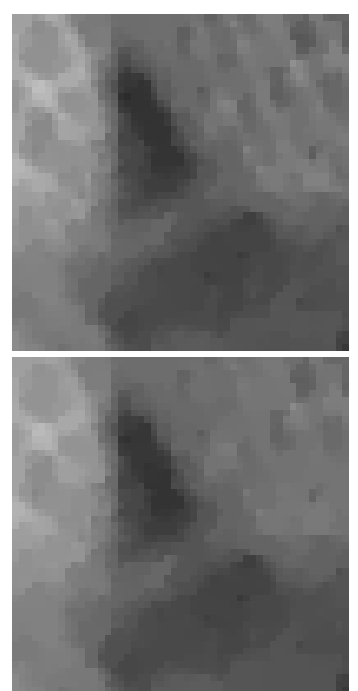

(c)

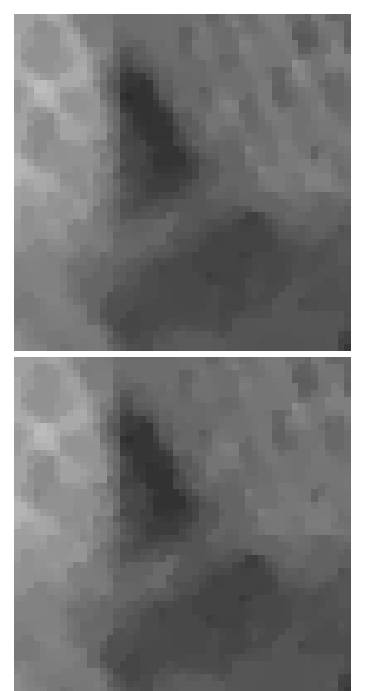

(d)

Figure 5. Comparison of zoomed fused images in Figure 4, where the white Gaussian noise $\sigma=20$ (in the 1st row) and $\sigma=30$ (in the 2nd row), respectively. (a) FSG [23]; (b) WTV [20]; (c) TVL1; (d) TVL2.

\section{References}

[1] R. S. Blum, Multi-Sensor Image Fusion and its Applications, (special series on Signal Processing and Communications). 2005.

[2] N. Burgos, M. J. Cardoso, M. Modat, S. Pedemonte, J. Dickson, A. Barnes, J. S. Duncan, D. Atkinson, S. R. Arridge, B. F. Hutton, and S. Ourselin, Attenuation Correction Synthesis for Hybrid PET-MR Scanners, pp. 147-154. Springer Berlin Heidelberg, 2013.

[3] A. Ardeshir Goshtasby and S. Nikolov, "Guest editorial: Image fusion: Advances in the state of the art," Information Fusion, vol. 8, no. 2, pp. 114-118, 2007.

[4] B. Yang and S. Li, "Pixel-level image fusion with simultaneous orthogonal matching pursuit," Information Fusion, vol. 13, no. 1, pp. 10-19, 2012.

[5] V. Tsagaris and V. Anastassopoulos, "Information measure for assessing pixel-level fusion methods," in Proceedings of SPIE, vol. 5573, pp. 64-71, 2004.

[6] N. Mitianoudis and T. Stathaki, "Pixel-based and region-based image fusion schemes using ICA bases," Information Fusion, vol. 8, no. 2, pp. 131-142, 2007.

[7] M. Kumar and S. Dass, "A total variation-based algorithm for pixel-level image fusion," IEEE Transactions on Image Processing, vol. 18, no. 9, pp. 2137-2143, 2009.

[8] S. Li and B. Yang, "Multifocus image fusion using region segmentation and spatial frequency," Image and Vision Computing, vol. 26, no. 7, pp. 971-979, 2008.

[9] P. Shah, S. N. Merchant, and U. B. Desai, "An efficient spatial domain fusion scheme for multifocus images using statistical properties of neighborhood," in 2011 IEEE International Conference on Multimedia and Expo, pp. 1-6, 2011. 


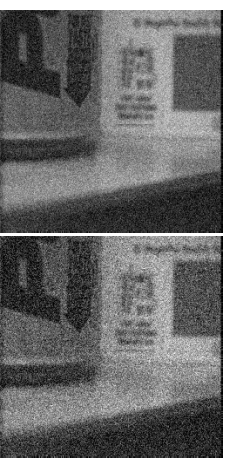

(a)

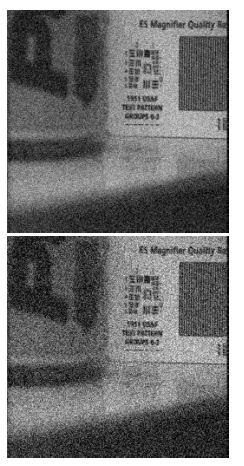

(b)

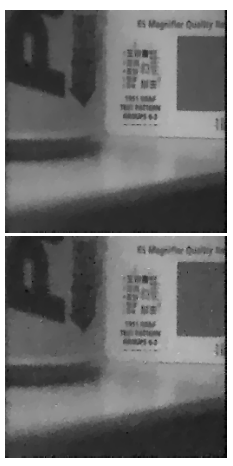

(c)

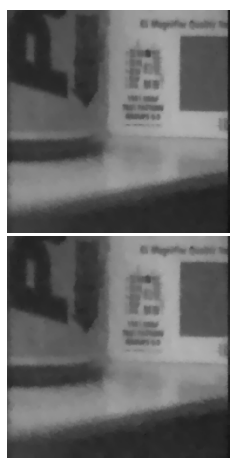

(d)

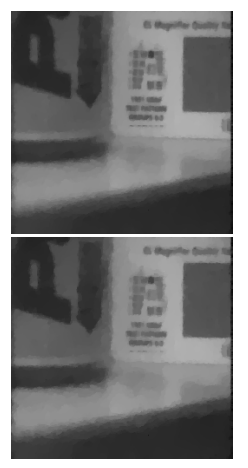

(e)

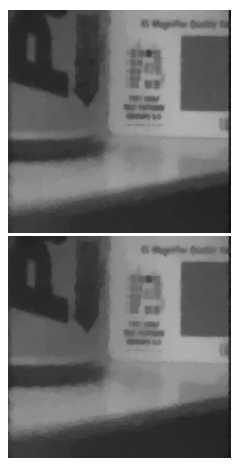

(f)

Figure 6. Comparison of different methods for the images "Book", where the white Gaussian noise $\sigma=20$ (in the 1st row) and $\sigma=30$ (in the 2nd row), respectively. (a) and (b): two input source images; (c) FSG [23]; (d) WTV [20]; (e) TVL1; (f) TVL2.

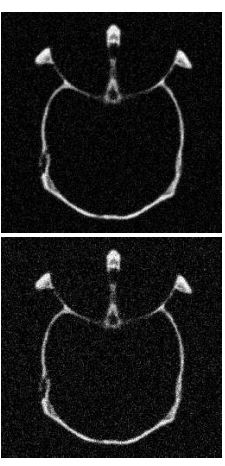

(a)

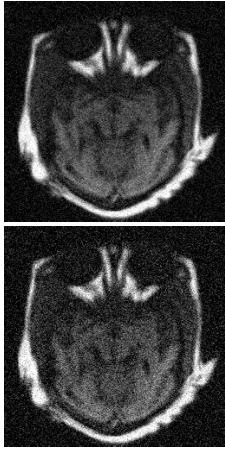

(b)

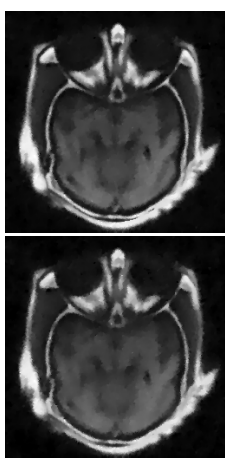

(c)

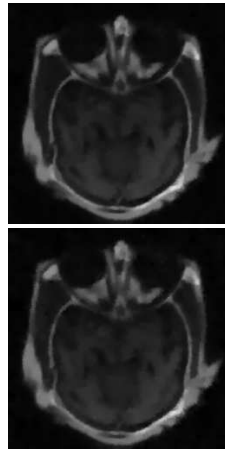

(d)

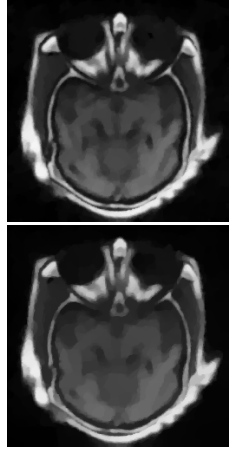

(e)

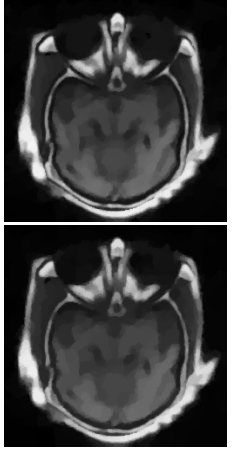

(f)

Figure 7. Comparison of different models for fusing and denoising in the images "Brain", where white Gaussian noise $\sigma=20$ (in the 1st row) and $\sigma=30$ (in the 2nd row), respectively. (a) and (b): two source images; (c) FSG [23]; (d) WTV [20]; (e) TVL1; (f) TVL2.

[10] S. Li, J. T. Kwok, and Y. Wang, "Combination of images with diverse focuses using the spatial frequency," Information Fusion, vol. 2, no. 3, pp. 169-176, 2001.

[11] G. Piella, "Image fusion for enhanced visualization: A variational approach," International Journal of Computer Vision, vol. 83, no. 1, pp. 1-11, 2009.

[12] D. A. Socolinsky and L. B. Wolff, "Multispectral image visualization through first-order fusion," IEEE Transactions on Image Processing, vol. 11, no. 8, pp. 923-931, 2002.

[13] A. Toet, "A morphological pyramidal image decomposition," Pattern Recognition Letters, vol. 9, no. 4, pp. 255-261, 1989.

[14] V. S. Petrovic and C. S. Xydeas, "Gradient-based multiresolution image fusion," IEEE Transactions on Image Processing, vol. 13, no. 2, pp. 228-237, 2004.

[15] H. Li, B. S. Manjunath, and S. K. Mitra, "Multi-sensor image fusion using the wavelet transform," in Proceedings of 1st International Conference on Image Processing, vol. 1, pp. 51$55,1994$. 
Table 3. The values of $Q_{M I}$ and $Q_{P}$ by using different methods for the real multi-modal images.

\begin{tabular}{|c|c|c|c|c|c|c|c|c|c|}
\hline \multirow[b]{2}{*}{ Image } & \multirow[b]{2}{*}{ Noise } & \multicolumn{4}{|c|}{$Q_{M I}$} & \multicolumn{4}{|c|}{$Q_{P}$} \\
\hline & & FSG & WTV & TVL1 & TVL2 & FSG & WTV & TVL1 & TVL2 \\
\hline \multirow{4}{*}{ Brain } & 15 & 2.7071 & 2.5786 & 2.7946 & 2.8031 & 0.2190 & 0.2014 & 0.2102 & 0.2140 \\
\hline & 20 & 2.5995 & 2.4550 & 2.6968 & 2.7032 & 0.1778 & 0.1698 & 0.1749 & 0.1783 \\
\hline & 25 & 2.5226 & 2.3498 & 2.6252 & 2.6268 & 0.1518 & 0.1481 & 0.1533 & 0.1572 \\
\hline & 30 & 2.4533 & 2.2695 & 2.5618 & 2.5801 & 0.1330 & 0.1314 & 0.1334 & 0.1404 \\
\hline \multirow{4}{*}{ Grass } & 15 & 3.1161 & 2.6442 & 3.1560 & 3.1621 & 0.4820 & 0.3601 & 0.4920 & 0.4941 \\
\hline & 20 & 3.0000 & 2.5556 & 3.0039 & 3.0102 & 0.4128 & 0.2943 & 0.4160 & 0.4195 \\
\hline & 25 & 2.8867 & 2.4823 & 2.8949 & 2.9018 & 0.3502 & 0.2449 & 0.3568 & 0.3594 \\
\hline & 30 & 2.7917 & 2.4173 & 2.7991 & 2.8035 & 0.2993 & 0.2094 & 0.3059 & 0.3091 \\
\hline \multirow{4}{*}{ Crossroad } & 15 & 2.7518 & 2.1029 & 2.8244 & 2.8381 & 0.2861 & 0.2531 & 0.2955 & 0.2987 \\
\hline & 20 & 2.5226 & 1.9897 & 2.6266 & 2.6408 & 0.2343 & 0.2185 & 0.2462 & 0.2507 \\
\hline & 25 & 2.3753 & 1.9043 & 2.4832 & 2.4976 & 0.2006 & 0.1916 & 0.2081 & 0.2150 \\
\hline & 30 & 2.2320 & 1.8284 & 2.3733 & 2.3950 & 0.1736 & 0.1709 & 0.1851 & 0.1888 \\
\hline \multirow{4}{*}{ Hill } & 15 & 2.5452 & 1.7110 & 2.6284 & 2.6367 & 0.1082 & 0.1091 & 0.1143 & 0.1166 \\
\hline & 20 & 2.3140 & 1.6892 & 2.4872 & 2.5016 & 0.0786 & 0.0925 & 0.0947 & 0.0962 \\
\hline & 25 & 2.2545 & 1.6677 & 2.3845 & 2.4061 & 0.0738 & 0.0806 & 0.0843 & 0.0887 \\
\hline & 30 & 2.1680 & 1.6477 & 2.3222 & 2.3709 & 0.0623 & 0.0724 & 0.0819 & 0.0897 \\
\hline \multirow{4}{*}{ Plant } & 15 & 3.3209 & 3.1157 & 3.3264 & 3.3287 & 0.4060 & 0.3734 & 0.4145 & 0.4128 \\
\hline & 20 & 3.1492 & 2.9502 & 3.1597 & 3.1608 & 0.3330 & 0.3122 & 0.3411 & 0.3411 \\
\hline & 25 & 3.0190 & 2.8258 & 3.0270 & 3.0318 & 0.2753 & 0.2553 & 0.2860 & 0.2858 \\
\hline & 30 & 2.8930 & 2.7230 & 2.9159 & 2.9197 & 0.2305 & 0.2197 & 0.2422 & 0.2420 \\
\hline
\end{tabular}

Table 4. The CPU times (in seconds) consumed by solving the four models.

\begin{tabular}{ccccccccc}
\hline & Leaves & Book & Plane & Brain & Grass & Crossroad & Hill & Plane \\
\hline FSG & 3.12 & 8.78 & 1.58 & 7.40 & 3.79 & 3.61 & 3.62 & 3.77 \\
WTV & 1.10 & 1.23 & 0.72 & 1.24 & 1.46 & 1.42 & 0.99 & 1.37 \\
TVL1 & 1.75 & 3.70 & 0.38 & 3.99 & 1.68 & 2.29 & 1.33 & 3.10 \\
TVL2 & 1.39 & 2.43 & 0.51 & 2.72 & 2.34 & 2.34 & 0.88 & 2.46 \\
\hline
\end{tabular}

[16] V. Naidu and J. Raol, "Pixel-level image fusion using wavelets and principal component analysis,” Defence Science Journal, vol. 58, no. 3, p. 338, 2008.

[17] F. Nencini, A. Garzelli, S. Baronti, and L. Alparone, "Remote sensing image fusion using the curvelet transform," Information Fusion, vol. 8, no. 2, pp. 143-156, 2007.

[18] Y. Chai, H. Li, and X. Zhang, "Multifocus image fusion based on features contrast of multiscale products in nonsubsampled contourlet transform domain," Optik-International Journal for Light and Electron Optics, vol. 123, no. 7, pp. 569-581, 2012.

[19] G. Guorong, X. Luping, and F. Dongzhu, "Multi-focus image fusion based on nonsubsampled shearlet transform," IET Image Processing, vol. 7, pp. 633-639, 2013.

[20] W. W. Wang, P. L. Shui, and X. C. Feng, "Variational models for fusion and denoising of multifocus images," IEEE Signal Processing Letters, vol. 15, pp. 65-68, 2008. 

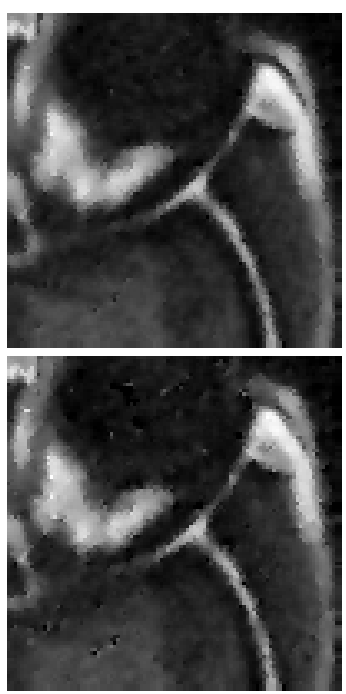

(a)
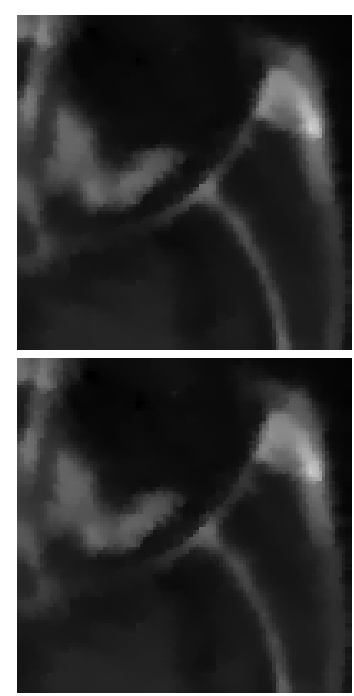

(b)
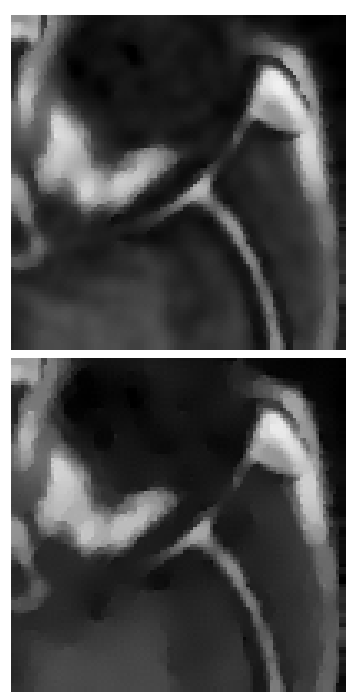

(c)
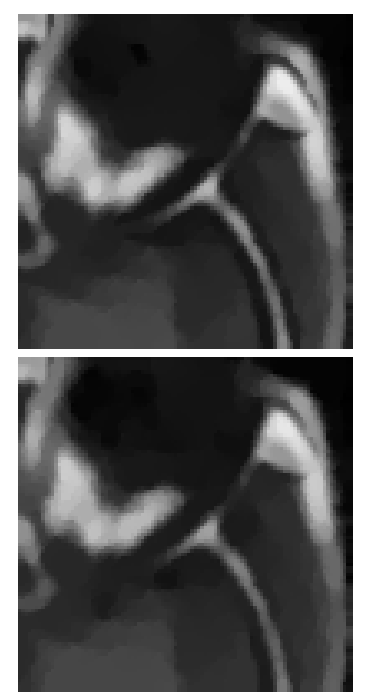

(d)

Figure 8. Comparison of zoomed fused images in Figure 7, where the white Gaussian noise $\sigma=20$ (in the 1st row) and $\sigma=30$ (in the 2nd row), respectively. (a) FSG [23]; (b) WTV [20]; (c) TVL1; (d) TVL2.

[21] B. Lu, C. Miao, and H. Wang, "Pixel level image fusion based on linear structure tensor," in 2010 IEEE Youth Conference on Information, Computing and Telecommunications, pp. 303$306,2010$.

[22] Z. Zhou, S. Li, and B. Wang, "Multi-scale weighted gradient-based fusion for multi-focus images," Information Fusion, vol. 20, pp. 60 - 72, 2014.

[23] F. Li and T. Zeng, "Variational image fusion with first and second-order gradient information," Journal of Computational Mathematics, vol. 34, no. 2, pp. 200-222, 2016.

[24] Y. Xu, T. Z. Huang, J. Liu, and X. G. Lv, "Split Bregman iteration algorithm for image deblurring using fourth-order total bounded variation regularization model," Journal of Applied Mathematics, vol. 2013, no. 3, pp. 417-433, 2013.

[25] T. Goldstein and S. Osher, "The split Bregman method for L1-regularized problems," SIAM Journal on Imaging Sciences, vol. 2, no. 2, pp. 323-343, 2009.

[26] J.-F. Cai, S. Osher, and Z. Shen, "Split Bregman methods and frame based image restoration," Multiscale Modeling \& Simulation, vol. 8, no. 2, pp. 337-369, 2010.

[27] L. Yu and W. Zengfu, "Simultaneous image fusion and denoising with adaptive sparse representation," IET Image Processing, vol. 9, pp. 347-357(10), 2015.

[28] J. Zhang and K. Chen, "A total fractional-order variation model for image restoration with nonhomogeneous boundary conditions and its numerical solution," SIAM Journal on Imaging Sciences, vol. 8, no. 4, pp. 2487-2518, 2015.

[29] J. Yang, Y. Zhang, and W. Yin, "A fast alternating direction method for TVL1-L2 signal reconstruction from partial Fourier data," IEEE Journal of Selected Topics in Signal Processing, vol. 4, no. 2, pp. 288-297, 2010. 


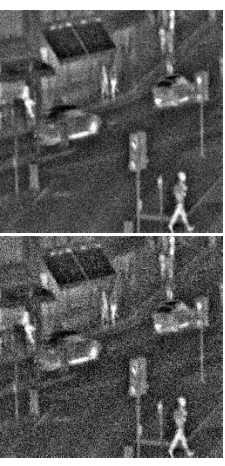

(a)

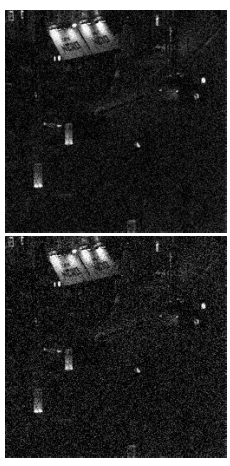

(b)

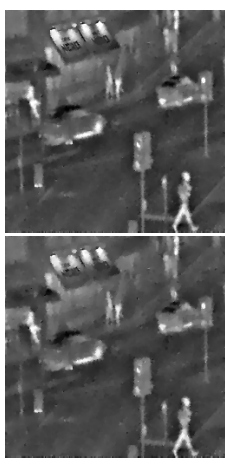

(c)

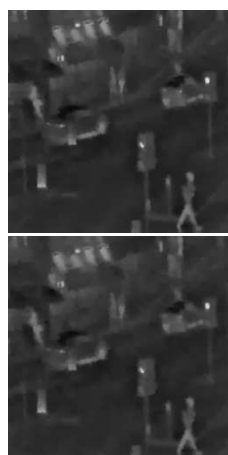

(d)

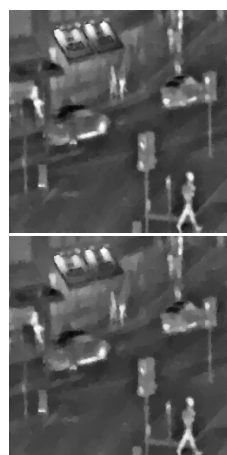

(e)

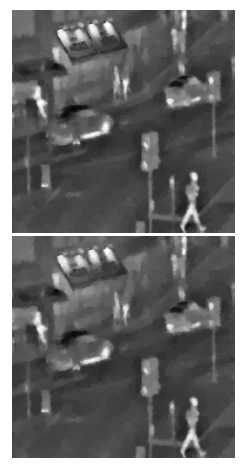

(f)

Figure 9. Comparison of different method for fusing and denoising in the image "Crossroad" with Gaussian white noise $\sigma=20$ (in the 1st row) and $\sigma=30$ (in the 2nd row), respectively. (a) and (b) two source images; (c)-(f) fused images by applying different methods: (c) FSG [23]; (d) WTV [20]; (e) TVL1; (f) TVL2.

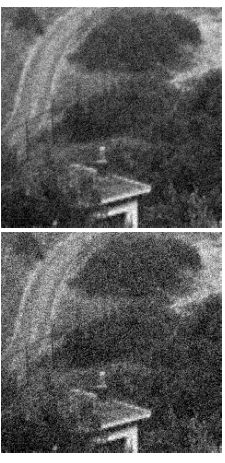

(a)

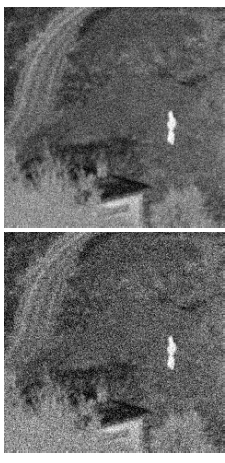

(b)

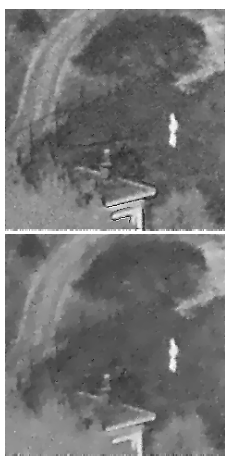

(c)

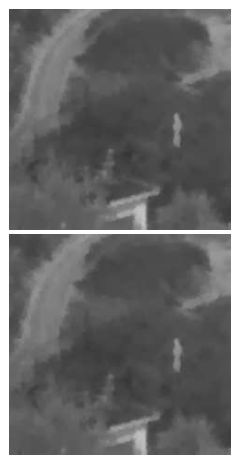

(d)

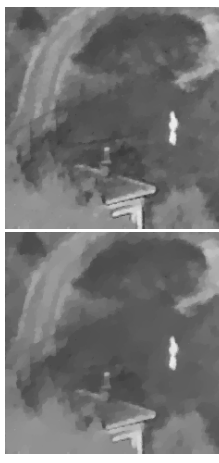

(e)

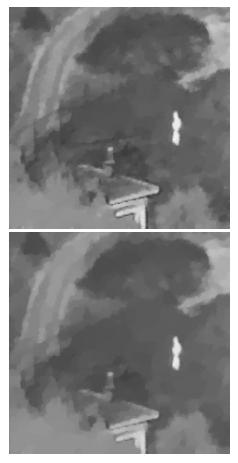

(f)

Figure 10. Comparison of different method for fusing and denoising in the image "Hill" with Gaussian white noise $\sigma=20$ (in the 1st row) and $\sigma=30$ (in the 2nd row), respectively. (a) and (b) two source images; (c)-(f) fused images by applying different methods: (c) FSG [23]; (d) WTV [20]; (e) TVL1; (f) TVL2.

[30] B. He and X. Yuan, "On the $\mathrm{O}(1 / \mathrm{n})$ convergence rate of the Douglas-Rachford alternating direction method," SIAM Journal on Numerical Analysis, vol. 50, no. 2, pp. 700-709, 2012.

[31] C. Chen, M. K. Ng, and X.-L. Zhao, "Alternating direction method of multipliers for nonlinear image restoration problems," IEEE Transactions on Image Processing, vol. 24, no. 1, pp. 33-43, 2015.

[32] Z. Ren, C. He, and Q. Zhang, "Fractional order total variation regularization for image superresolution," Signal Processing, vol. 93, no. 9, pp. 2408 - 2421, 2013.

[33] J. Zhang, Z. Wei, and L. Xiao, "A fast adaptive reweighted residual-feedback iterative algorithm for fractional-order total variation regularized multiplicative noise removal of partlytextured images," Signal Processing, vol. 98, pp. 381 - 395, 2014.

[34] Y. Zhang, W. Zhang, Y. Lei, and J. Zhou, "Few-view image reconstruction with fractionalorder total variation," J. Opt. Soc. Am. A, vol. 31, no. 5, pp. 981-995, 2014. 

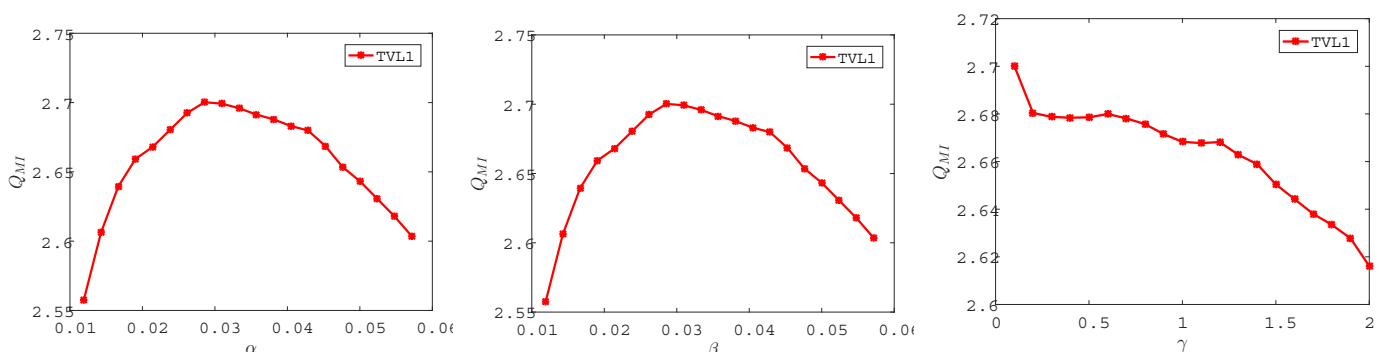

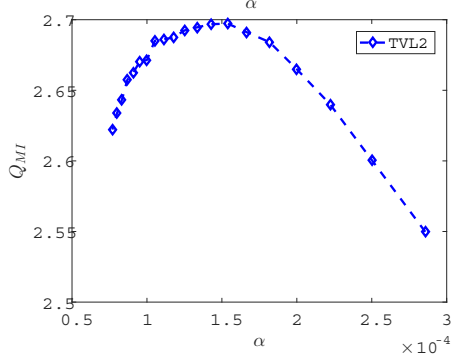

(a)

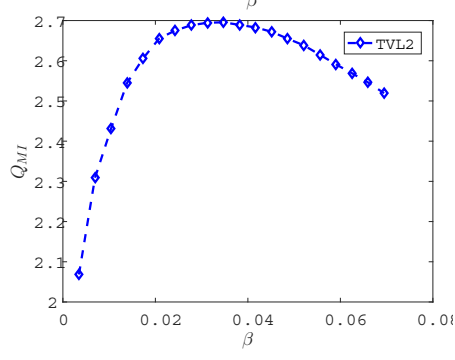

(b)

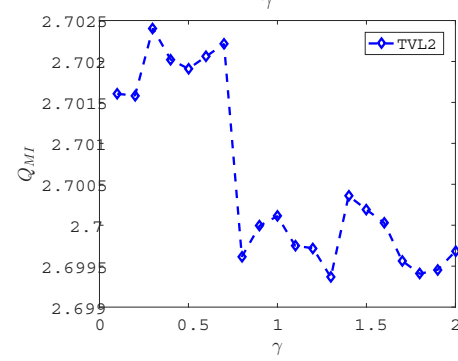

(c)

Figure 11. The values of $Q_{M I}$ for the fused images obtained by our methods for different parameters. (a) Different $\alpha$; (b) different $\beta$; (c) different $\gamma$.

[35] I. Podlubny, Fractional differential equations: an introduction to fractional derivatives, fractional differential equations, to methods of their solution and some of their applications, vol. 198. Academic press, 1998.

[36] K. S. Miller and B. Ross, An Introduction to the Fractional Calculus and Fractional Differential Equations. Wiley-Interscience, 1 ed., 1993.

[37] K. Oldham and J. Spanier, The fractional calculus theory and applications of differentiation and integration to arbitrary order, vol. 111. Elsevier, 1974.

[38] A. A. Kilbas, H. M. Srivastava, and J. J. Trujillo, Theory and Applications of Fractional Differential Equations, Volume 204 (North-Holland Mathematics Studies). New York, NY, USA: Elsevier Science Inc., 2006.

[39] I. Podlubny, A. Chechkin, T. Skovranek, Y. Chen, and B. M. V. Jara, "Matrix approach to discrete fractional calculus ii: Partial fractional differential equations," Journal of Computational Physics, vol. 228, no. 8, pp. 3137 - 3153, 2009.

[40] H. Wang and N. Du, "Fast solution methods for space-fractional diffusion equations," Journal of Computational and Applied Mathematics, vol. 255, pp. 376 - 383, 2014.

[41] L. I. Rudin, S. Osher, and E. Fatemi, "Nonlinear total variation based noise removal algorithms," Physica D: Nonlinear Phenomena, vol. 60, no. 1, pp. 259-268, 1992.

[42] G. Pajares and J. M. de la Cruz, "A wavelet-based image fusion tutorial," Pattern Recognition, vol. 37, no. 9, pp. 1855 - 1872, 2004.

[43] Q. Zhang and B. long Guo, "Multifocus image fusion using the nonsubsampled contourlet transform," Signal Processing, vol. 89, no. 7, pp. 1334 - 1346, 2009. 
[44] J.-J. Mei and T.-Z. Huang, "Primal-dual splitting method for high-order model with application to image restoration," Applied Mathematical Modelling, vol. 40, no. 3, pp. 2322 - 2332, 2016.

[45] A. Chambolle and T. Pock, "A first-order primal-dual algorithm for convex problems withapplications to imaging," Journal of Mathematical Imaging and Vision, vol. 40, no. 1, pp. 120 $145,2011$.

[46] Y. Wang, J. Yang, W. Yin, and Y. Zhang, "A new alternating minimization algorithm for total variation image reconstruction," SIAM Journal on Imaging Sciences, vol. 1, no. 3, pp. 248$272,2008$.

[47] X.-L. Zhao, W. Wang, T.-Y. Zeng, T.-Z. Huang, and M. K. Ng, "Total variation structured total least squares method for image restoration," SIAM Journal on Scientific Computing, vol. 35, no. 6, pp. B1304-B1320, 2013.

[48] T.-H. Ma, T.-Z. Huang, X.-L. Zhao, and Y. Lou, "Image deblurring with an inaccurate blur kernel using a group-based low-rank image prior," Information Sciences, vol. 408, pp. 213 233, 2017.

[49] X.-L. Zhao, F. Wang, and M. K. Ng, "A new convex optimization model for multiplicative noise and blur removal," SIAM Journal on Imaging Sciences, vol. 7, no. 1, pp. 456-475, 2014.

[50] J.-J. Mei, Y. Dong, T.-Z. Huang, and W. Yin, "Cauchy noise removal by nonconvex admm with convergence guarantees," Journal of Scientific Computing (online), May 2017.

[51] M. K. Ng, F. Wang, and X. Yuan, "Inexact alternating direction methods for image recovery," SIAM Journal on Scientific Computing, vol. 33, no. 4, pp. 1643-1668, 2011.

[52] M. R. Hestenes and E. Stiefel, Methods of conjugate gradients for solving linear systems, vol. 49. Journal of Research of the National Bureau of Standards, 1952.

[53] Glowinski and Roland, Numerical Methods for Nonlinear Variational Problems. SpringerVerlag, 1984.

[54] W. Y. Wei Deng and Y. Zhang, "Group sparse optimization by alternating direction method," in Proceedings of SPIE, vol. 8858, pp. 1-15, 2013.

[55] Z. Liu, E. Blasch, Z. Xue, J. Zhao, R. Laganiere, and W. Wu, "Objective assessment of multiresolution image fusion algorithms for context enhancement in night vision: A comparative study," IEEE Transactions on Pattern Analysis and Machine Intelligence, vol. 34, pp. 94-109, Jan 2012.

[56] G. Qu, D. Zhang, and P. Yan, "Information measure for performance of image fusion," Electronics Letters, vol. 38, no. 7, pp. 313-315, 2002.

[57] M. Hossny, S. Nahavandi, and D. Creighton, "Comments on'information measure for performance of image fusion'," Electronics letters, vol. 44, no. 18, pp. 1066-1067, 2008.

[58] J. Zhao, R. Laganiere, and Z. Liu, "Performance assessment of combinative pixel-level image fusion based on an absolute feature measurement," International Journal of Innovative Computing, Information and Control, vol. 3, no. 6, pp. 1433-1447, 2007. 
[59] Z. Liu, D. S. Forsyth, and R. Laganiere, "A feature-based metric for the quantitative evaluation of pixel-level image fusion," Computer Vision and Image Understanding, vol. 109, no. 1 , pp. $56-68,2008$. 